\title{
Biodegradación de contaminantes orgánicos de la industria láctea
}

\author{
(Biodegradation of organic contaminants from the \\ dairy industry)
}

\author{
Diego Prócel ${ }^{1}$, Paola Posligua', Carlos Banchón ${ }^{1}$
}

\begin{abstract}
Resumen:
Uno de los aspectos medioambientales de alto impacto en las industrias lácteas es la descarga de aguas residuales, tanto por la materia orgánica contaminante como por la considerable generación de efluentes. En el presente estudio, se implementó la biofiltración de efluentes de una lechera en San Miguel de Nono (Ecuador) con carga contaminante de $10.000 \mathrm{mg} / \mathrm{L}$ en términos de demanda química de oxígeno y turbidez de 799 NTU. El sistema con una capacidad de $55 \mathrm{~L}$ por cada lote consistió de tres biofiltros en serie y clarificación asistida con zeolita activada. La degradación orgánica alcanzó porcentajes de 98,9\%; la remoción de turbidez fue de $95,2 \%$, de nitrógeno $94,4 \%$ y de fósforo $89,1 \%$. La presencia de grasa de leche no disminuyó la eficiencia de la biofiltración sino que incrementó la remoción de materia suspendida y neutralización del pH en la clarificación. El tiempo óptimo de operación fue de 6 horas bajo condiciones aerobias.
\end{abstract}

Palabras clave: biofiltración; biopelícula; zeolita; industria láctea; oxidación

\begin{abstract}
:
One of the environmental aspects of high impact in dairy industries is the discharge of wastewaters, because of contaminating organic matter as well as the substantial generation of effluents. In the present study, a biofiltration of effluents from a dairy industry in San Miguel de Nono (Ecuador) with a pollution load of $10.000 \mathrm{mg} / \mathrm{L}$ in terms of chemical oxygen demand and turbidity of 799 NTU was implemented. The system with a capacity of $55 \mathrm{~L}$ per batch consisted of three biofilters in series and activated zeolite assisted clarification. Organic degradation reached $98,9 \%$; turbidity removal was $95,2 \%, 94,4 \%$ of nitrogen and $89,1 \%$ of phosphorus. The presence of milk fat did not decrease the efficiency of the biofiltration but increased the removal of suspended matter and $\mathrm{pH}$ neutralisation in the clarification. The optimal operating time was 6 hours under aerobic conditions.
\end{abstract}

Keywords: biofiltration; biofilm; zeolite; dairy industry; oxidation

\footnotetext{
${ }^{1}$ Universidad de Las Américas, Quito - Ecuador (\{daprocel, p.posligua, c.banchon\} @udlanet.ec)
} 


\section{Introducción}

La industria láctea por cada litro de leche procesada genera hasta $10 \mathrm{~L}$ de agua residual (Porwal, Mane, \& Velhal, 2015). Tomando en cuenta que entre 1982 y 2012, esta industria a nivel mundial incrementó su producción de 482 a 754 millones de toneladas, es de gran interés el impacto ambiental que ésta genera (Food and Agriculture Organization of the United Nations, 2014); por ejemplo, en India se descargan anualmente 200 millones de toneladas de efluentes (Dawood, Kumar, \& Sambi, 2011). En Ecuador se desconoce informaciones sobre el monitoreo de efluentes de industrias lácteas a cuerpos naturales de agua, aunque se reporta una producción nacional de aproximadamente dos millones de litros diarios de leche, con un crecimiento de hasta $30 \%$ anual en el consumo (Ministerio de Industrias y Productividad, 2014). De alta preocupación es el efecto nocivo para la biodiversidad provocado por efluentes de lecheras, puesto que contienen altas concentraciones de materia orgánica por encima de los $10000 \mathrm{mg} \cdot \mathrm{L}^{-1}$ así como también desechos inorgánicos como detergentes y sanitizantes (Verheijen, 1996; Carvalho, Prazeres, \& Rivas, 2013; Porwal, Mane, \& Velhal, 2015).

Degradación de la contaminación con eficiencias por encima del 90\% e incluso con producción de energía renovable se ha logrado con procesos biológicos aerobios-anaerobios (lodos activados, lagunas de oxidación, biofiltros, reactores en secuencia) y procesos químicos tales como coagulación-floculación, ya sea como flotación por aire disuelto o electrocoagulación (Karadag et al., 2015; Porwal, Mane, \& Velhal, 2015). Sin embargo, los altos costos de operación y alta producción de lodos residuales se convierten en limitantes para la implementación de plantas de tratamiento en pequeñas industrias (Sirianuntapiboon, Jeeyachok \& Larplai, 2005). Por el contrario, de gran ventaja es la aplicación de filtros percoladores o biofiltros. Estos utilizan microorganismos inmovilizados que forman biopelículas las cuales evitan la generación de altos volúmenes de lodos, bajo consumo de energía, bajos tiempos de operación y altos grados de remoción de contaminación orgánica a temperaturas ambientales (Naz, Saroj, Mumtaz, Ali \& Ahmed, 2015). Por tanto, el tratamiento de aguas residuales basado en biofiltros es una alternativa económicamente amigable no solo con la pequeña industria sino también con el medio ambiente.

El tratamiento de agua residual mediante microorganismos inmovilizados (y como consecuencia, la formación de biopelículas) conlleva a la eficiente degradación de materia orgánica suspendida y disuelta. Es por ello que en el presente trabajo, se evaluaron las condiciones operacionales de un sistema de biofiltración para la biodegradación de materia orgánica suspendida y disuelta. 


\section{Metodología}

\subsection{Calidad del agua}

Se midió cada dos horas la turbidez del agua mediante nefelometría (marca HANNA, HI 88713, USA). El pH fue medido en potenciómetro (marca HANNA, HI 2550, USA) así mismo cada dos horas. La demanda química de oxígeno (DQO) en $\mathrm{mg} \mathrm{O}_{2} \mathrm{~L}^{-1}$ fue determinada mediante espectrofotometría UV-VIS a $620 \mathrm{~nm}$ de acuerdo con el decrecimiento de la concentración de cromato (Macherey-Nagel, Alemania). La determinación respirométrica de la demanda bioquímica de oxígeno $\left(\mathrm{DBO}_{5}\right)$ se realizó mediante el cambio de presión en microcosmos a $25 \stackrel{\circ}{\circ} \mathrm{C}$ durante 5 días, generado por la degradación de materia orgánica en muestras de agua según el método descrito por WTW (Weilheim, Alemania). Las concentraciones de nitrógeno total y fósforo fueron determinadas mediante espectrofotometría (métodos analíticos estándar CP-PEE-A007/A008 del laboratorio CESAQ-PUCE). En total, $360 \mathrm{~L}$ de agua residual fueron recogidos de la descarga final de una industria láctea de San Miguel de Nono (Distrito Metropolitano de Quito, Ecuador) durante un periodo de 4 meses. El agua residual fue sometida a un desbaste para eliminación de material grueso antes de su remediación en el sistema de biofiltros.

\subsection{Aislamiento y crecimiento de microorganismos degradadores}

Las muestras de agua residual de la industria lechera fueron filtradas en filtro de papel Whatman (187 g.m ${ }^{-2}$ ) previa inoculación en agar nutritivo (Merck, Alemania) durante 1 día a $37^{\circ} \mathrm{C}$. Muestras de colonias del medio sólido luego fueron transferidas a un caldo nutritivo a $37^{\circ} \mathrm{C}$ bajo agitación continua a 200 rpm. Posteriormente, se procedió a inmovilizar microorganismos en soportes de plástico y fibra orgánica (500 mL medio con microorganismos por $20 \mathrm{~g}$ de soportes plásticos) a $37^{\circ} \mathrm{C}$ durante 72 horas y agitación continua a $200 \mathrm{rpm}$. El crecimiento de la biopelícula fue optimizado con la adición controlada de una solución de fosfato sódico al $10 \%$.

\subsection{Sistema de biofiltración}

El sistema de biofiltración (Figura 1) consistió de tres etapas: (i) sistema de almacenamiento con aeración y distribución de agua residual, (ii) sistema de tres biofiltros en serie con microorganismos inmovilizados y (iii) sedimentación secundaria mediante zeolita activada. Desde el tanque de almacenamiento $\left(T_{1}\right)$ se distribuyeron $55 \mathrm{~L}$ de agua residual al sistema de biofiltros (R1, R2, R3) mediante bomba centrífuga. Cada lote de agua residual fue tratado en un tiempo máximo de 18 horas con mediciones de parámetros de calidad cada dos horas. La calidad del agua tratada mediante biofiltración solo fue medida en el biofiltro R3. El caudal de entrada del agua residual al biofiltro $\mathrm{R} 1$ fue de 3,5 L. $\mathrm{min}^{-1}$ y el de salida en el biofiltro R3 fue de 1,5 L. $\mathrm{min}^{-1}$. La temperatura promedio de trabajo fue $30^{\circ} \mathrm{C}$ y ésta se alcanzó con la ayuda de aislantes térmicos colocados en los biofiltros. La activación de la zeolita (Zeonatec, Ecuador) del tipo alumino-silicato 
de elevada hidratación se realizó a $250{ }^{\circ} \mathrm{C}$ durante cuatro horas con lavados constantes con agua destilada para eliminación de partículas contaminantes.

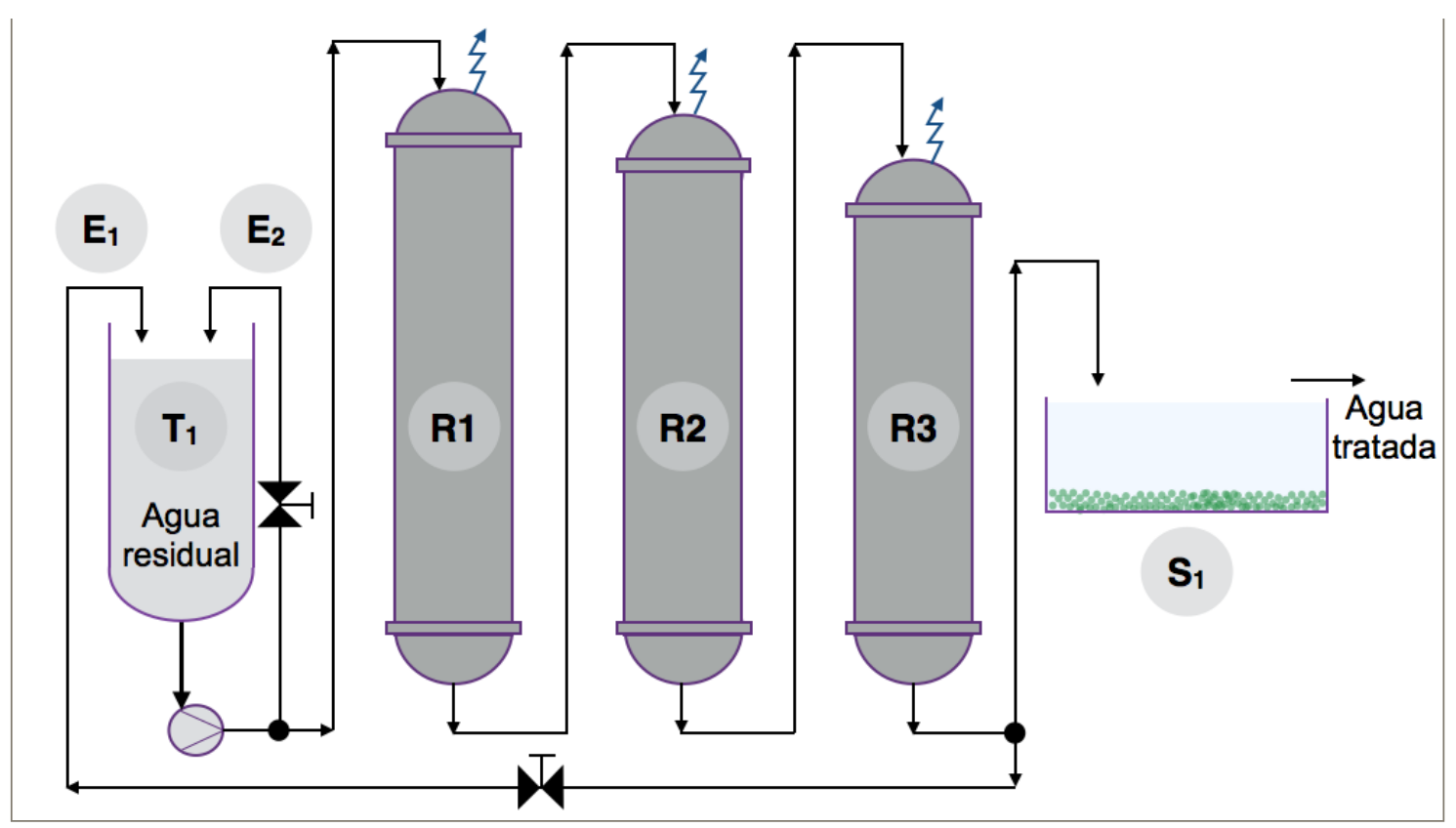

Figura 1. Diagrama del proceso de biofiltración de agua residual proveniente de una industria lechera. Donde $T_{1}=$ tanque de almacenamiento y aeración, $E_{1}=$ recirculación de agua tratada; $E_{2}=$ Recirculación de bomba centrífuga para control de caudal; R1,2,3 = biofiltros conectados en serie (4" de diámetro externo);

$\mathrm{S}_{1}=$ Sedimentación secundaria con zeolita activada.

\section{Resultados y Discusión}

Los resultados del proceso de tratamiento mediante biofiltración de las Figuras 2 y 3 representan 120 mediciones de un total de $360 \mathrm{~L}$ de agua residual tratada. Los cambios de $\mathrm{pH}$ y turbidez fueron medidos en función de la presencia de grasa en un lapso de 18 horas de monitoreo continuo. En experimentos preliminares se determinó que la presencia de grasa en el agua residual tuvo un efecto positivo en la eficiencia del tratamiento. Por tanto, se evaluó su influencia tanto en la remoción de turbidez como en el cambio de $\mathrm{pH}$ a la salida del biofiltro R3 y después de la sedimentación secundaria con zeolita activada.

Los resultados de la Fig. 2 indican que la remoción de grasa del agua residual tuvo como efecto que el pH se mantenga en niveles ácidos tanto a la salida de la biofiltración, como al final de la sedimentación secundaria después de 18 horas de monitoreo. También según la Fig. 2, la presencia o ausencia inicial de grasa generó en ambos casos el mismo efecto a la salida del sistema de biofiltración: el pH se mantuvo entre 4 y 5,5 durante 18 horas de monitoreo. Por otro lado, cuando se experimentó con la presencia de grasa en el agua residual, en la sedimentación con zeolita el pH del agua tratada fue 7 a partir de las 6 horas de tratamiento y se mantuvo así hasta las 18 horas de monitoreo. Es decir, la presencia inicial de grasa permitió que la sedimentación secundaria fuese más eficiente en la estabilización del pH. Es de notar que la 
presencia de sanitizantes en el agua residual de lecheras mejora la biodisponibilidad de los ácidos grasos de la leche en el medio líquido para la biodegradación microbiana. Los compuestos químicos tensoactivos por tanto generan un efecto surfactante que permite la disolución de grasas de la leche en el agua residual hasta el punto de promover la formación de biopelículas microbianas y flóculos de mayor tamaño que luego fueron fácilmente sedimentados en la etapa final de clarificación. Como consecuencia, los cambios de $\mathrm{pH}$ en el tratamiento con la presencia de grasa evidenciarían que efectos tensoactivos permiten alcanzar la estabilidad final del $\mathrm{pH}$.

Para los tratamientos con presencia/ausencia de grasas (Figura 2), los niveles de acidez del agua tratada en el sistema de biofiltración indican que no se completó un proceso de metanización, en el cual generalmente se alcanza un pH neutro. En la producción de metano, descensos de pH son relacionados con la producción y consumo de ácidos grasos volátiles bajo condiciones anaerobias, pero esta reacción se ve afectada si acaso los ácidos grasos no son convertidos en ácido acético por bacterias acetogénicas (De Mes, Stams, Reith \& Zeeman, 2003; Karadag et al., 2015). Es decir, los resultados indicarían que en la biofiltración no se efectuó la degradación completa y remoción de ácidos grasos (mayormente ácidos palmítico, esteárico y butírico) o ácidos orgánicos (p.e. ácido láctico).

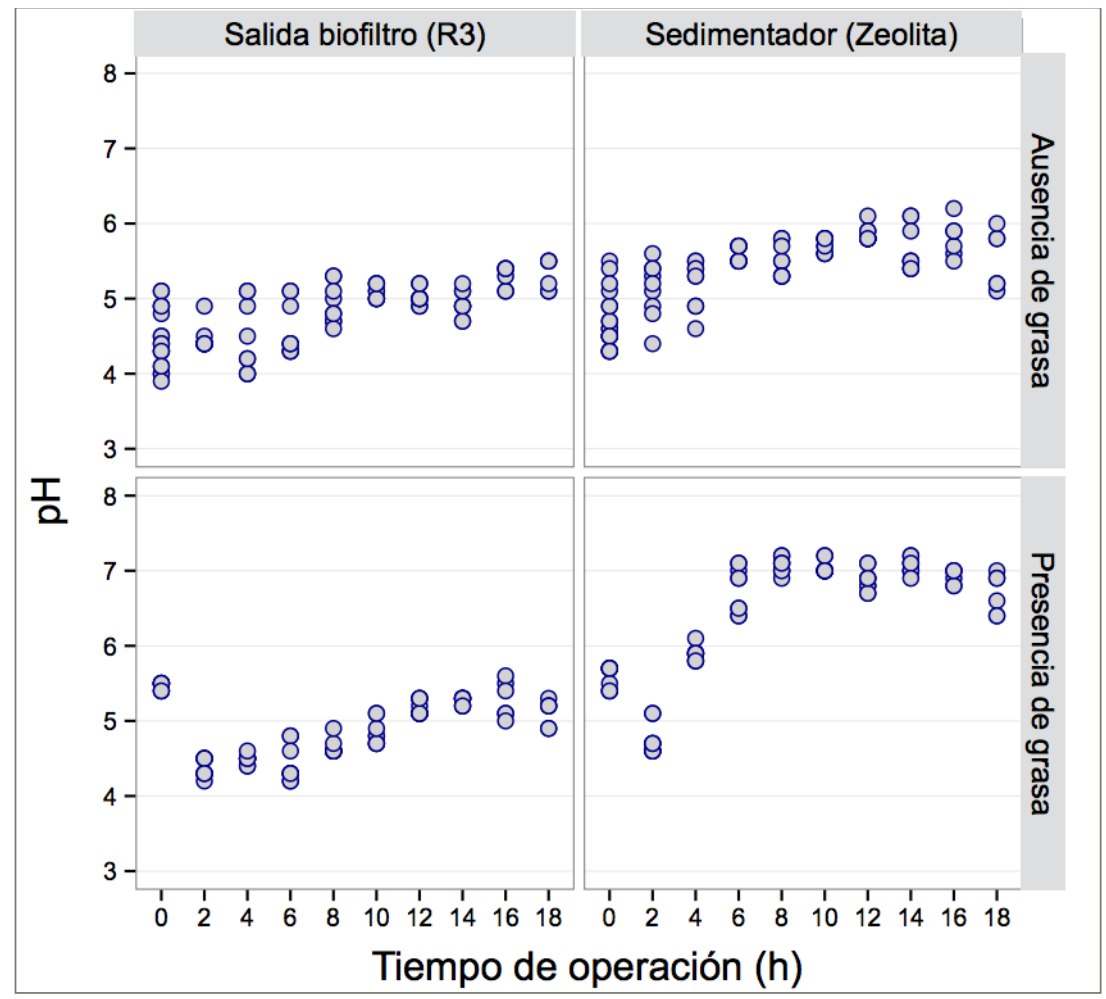

Figura 2. Efecto de la presencia/ausencia de grasa en el cambio de $\mathrm{pH}$ durante 18 horas de monitoreo. Mediciones a la salida del biofiltro R3 y después de la sedimentación con zeolita activada.

La leche contiene aproximadamente 400 tipos de ácidos grasos y en total la concentración de lípidos en la leche es de un 4,2\% (Månsson, 2008). Sobre la influencia de los lípidos en el proceso de remediación biológica, se ha reportado que estos en general inhiben tratamientos anaerobios y generan problemas operacionales en la transferencia de masa, no así en procesos aerobios 
(Demirel, Yenigun \& Onay, 2005; Karadag et al., 2015; Daverey \& Pakshirajan, 2015). Una de las razones para la dificultad en la degradación de lípidos radica en su baja biodisponibilidad, la cual en el presente estudio no se afectó debido al uso de biopelículas en el sistema de biofiltración. En concordancia con esto, los resultados de turbidez (Figura 3) evidencian que la presencia/ausencia de grasas no inhibe la biodegradación de la materia orgánica; por el contrario, en el caso de la presencia de grasas, aumenta el grado de sedimentación secundaria con zeolita activada. La capacidad de microorganismos en degradar fases lipídicas y disminución de la turbidez del medio hasta un $53 \%$ durante $48 \mathrm{~h}$ de tratamiento ha sido demostrada también en otro estudio (Porwal, Mane, \& Velhal, 2015).

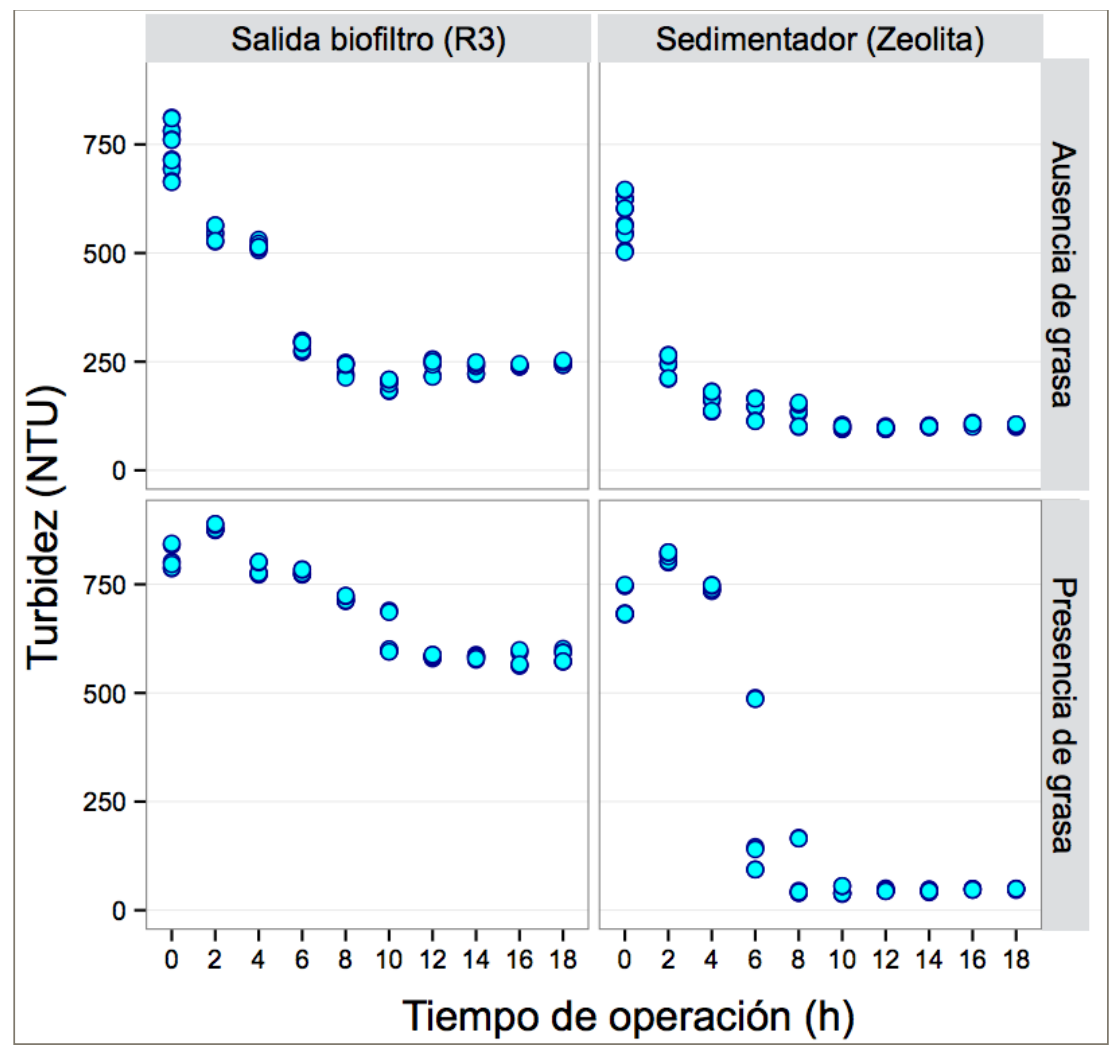

Figura 3. Efecto de la presencia de grasa en el cambio de turbidez final (NTU) durante 18 horas de tratamiento. Mediciones a la salida del biofiltro R3 y después de la sedimentación con zeolita activada.

Según la Figura 4, en presencia de grasa se alcanzan porcentajes de remoción de turbidez de $82,5 \%$ a las 6 horas y de $95,2 \%$ a las 10 horas de tratamiento. En presencia de grasa, la sedimentación secundaria sobrepasa el $90 \%$ de remoción de turbidez. En tratamientos en ausencia de grasa en el agua residual, se removió un $81,7 \%$ de turbidez a las 6 horas y $88,1 \%$ a las 10 horas; a las 18 horas se removió un $87,2 \%$ de turbidez. Sin grasa, el agua tratada no sobrepasó el $90 \%$ de remoción de turbidez. Aproximadamente 7 unidades de diferencia porcentual existe entre el tratamiento que utiliza grasa en la biofiltración y el tratamiento que no. Cabe recalcar que duramente 4 meses de experimentación, no existieron problemas de obstrucción en los biofiltros debido a los tratamientos que incluyeron grasas en el agua residual. Aparentemente, existe una degradación aerobia de lípidos que promueve la estabilización del pH y mejor calidad de flóculos sedimentables. 


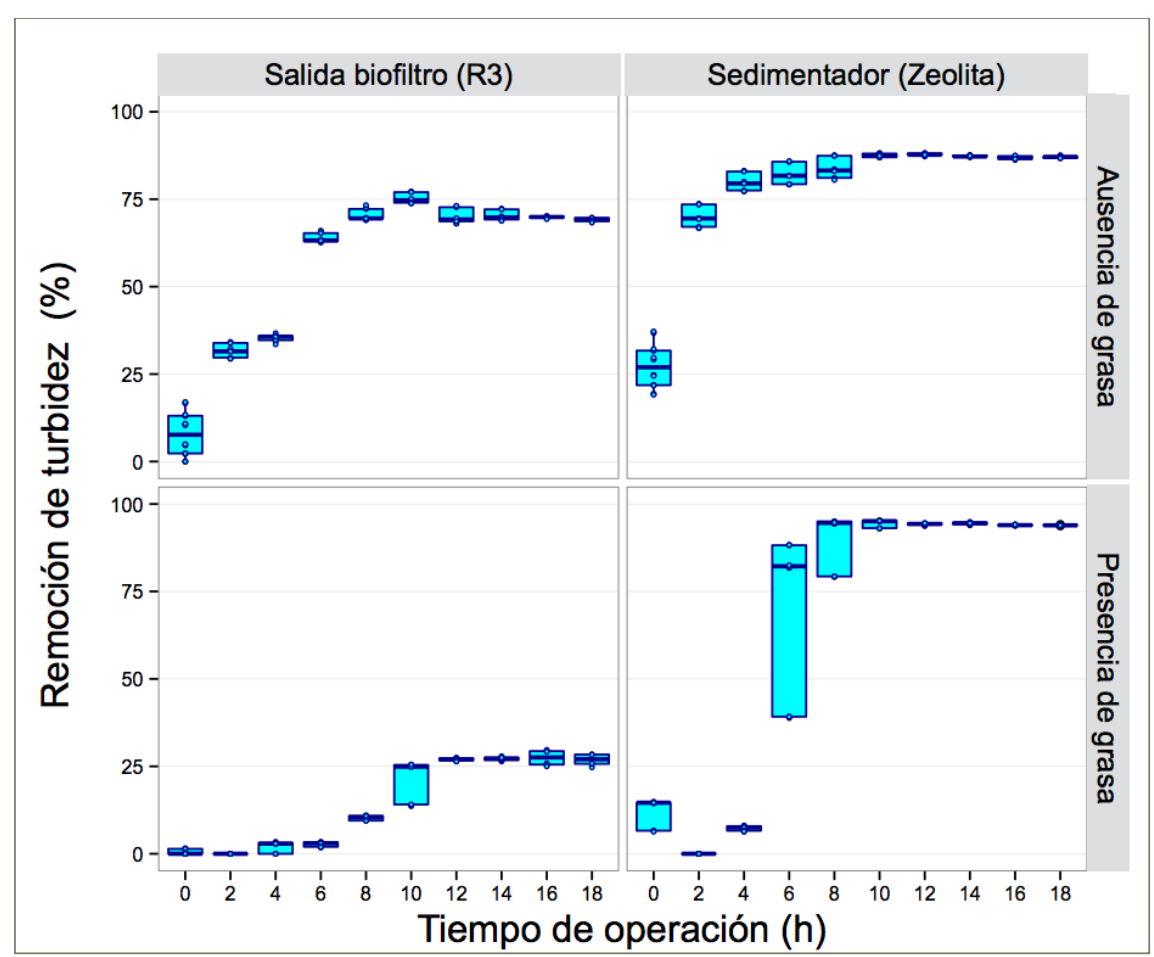

Figura 4. Porcentaje de remoción de turbidez en la salida del biofiltro R3 y después de la sedimentación con zeolita activada en presencia-ausencia de grasa durante 18 horas de operación.

Según los modelos matemáticos de las Figuras 5 y $6\left(R^{2}>0,92\right.$ y $\left.p<0,05\right)$, con presencia de grasa en el agua residual, 6 horas fue el tiempo mínimo de biofiltración lo cual generó una óptima sedimentación. Altas remociones de turbidez se alcanzaron cuando el pH fue neutro.

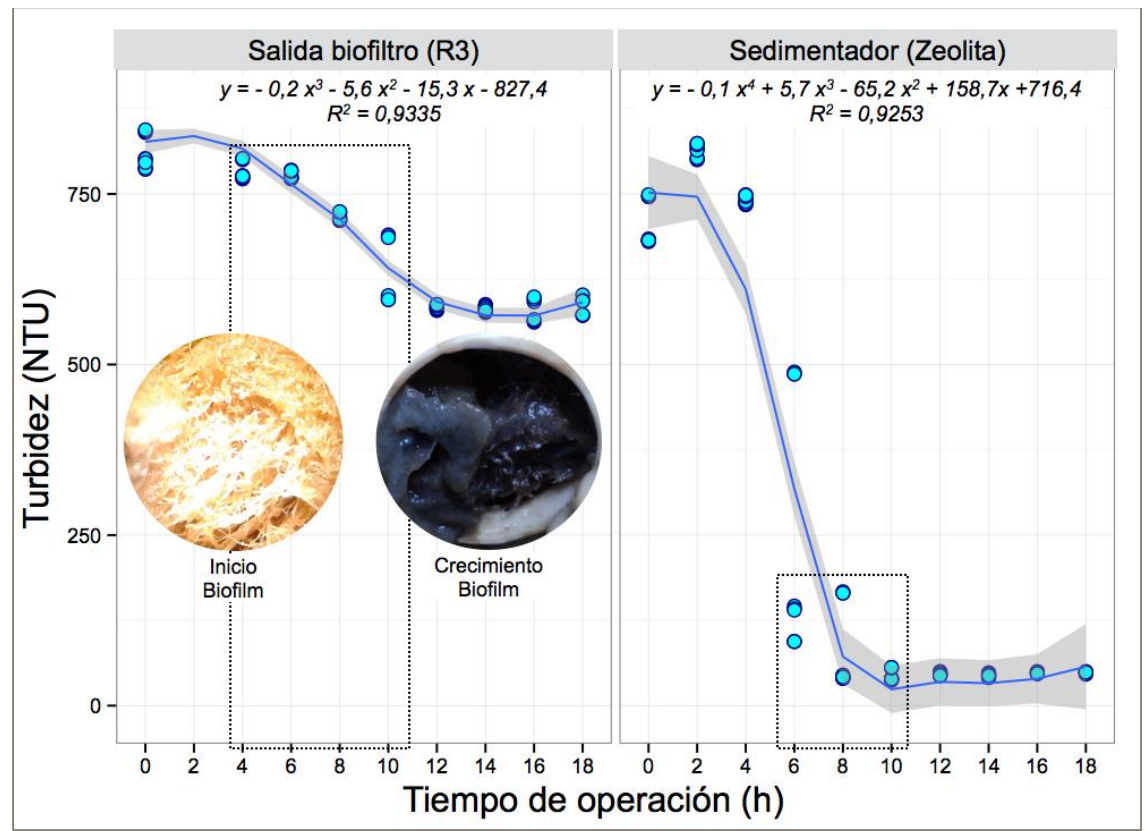

Figura 5. Modelo matemático para el cambio de turbidez en la salida del biofiltro R3 y después de la sedimentación con zeolita activada en presencia de grasa durante 18 horas de monitoreo. 


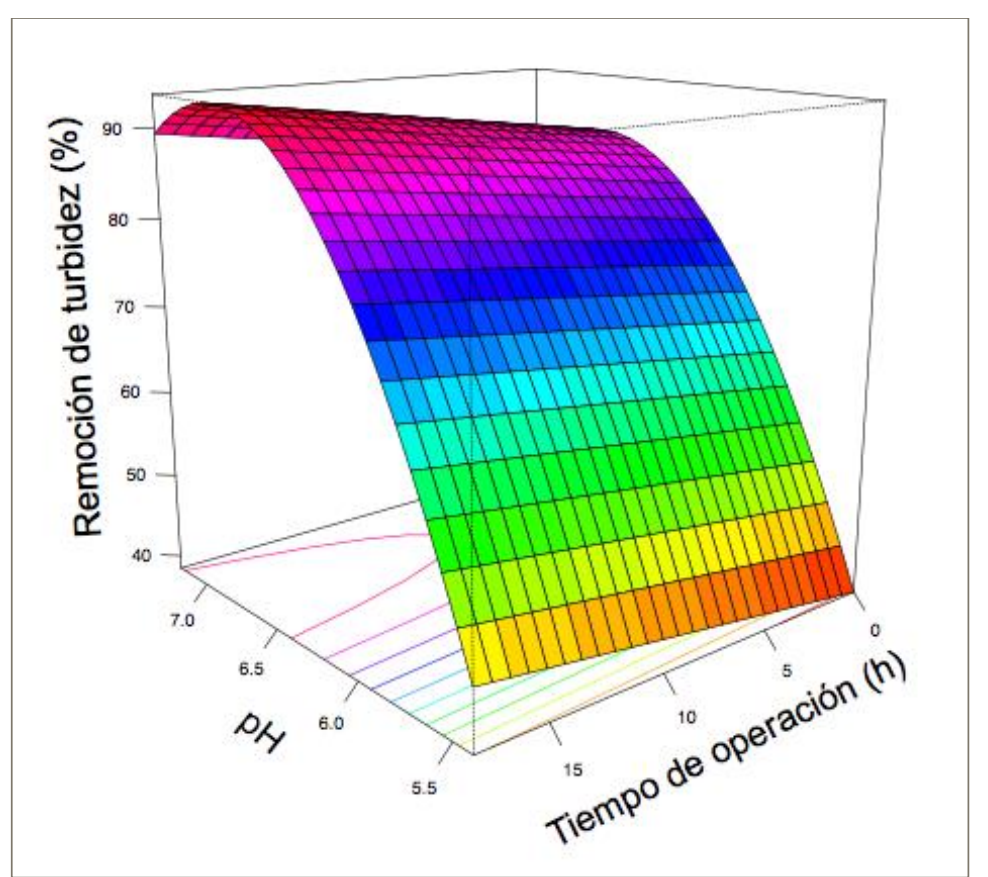

Figura 6. Modelo matemático del porcentaje de remoción de turbidez en función del cambio del pH, temperatura y tiempo de operación en la biofiltración de agua residual en presencia de grasa. Modelo desarrollado con el paquete RSM en R-project (R Core Team, 2014; Russell, 2014).

De acuerdo con los resultados obtenidos en las Figuras 2 y 3, del mejor tratamiento se evaluó la calidad del agua tratada a las 6 horas de operación, con presencia inicial de grasa y muestra tomada después de la sedimentación con zeolita activada (Figura 7). Se obtuvo una reducción de 98,9\% de DQO, 99,1\% de $\mathrm{DBO}_{5}, 95,2 \%$ en remoción de turbidez, 94,4\% en remoción de nitrógeno total y $89,1 \%$ en remoción de fósforo total. Todos los parámetros están por debajo de más del $90 \%$ de la normativa legal tanto ecuatoriana (Ministerio del Ambiente, 2008) como normativa internacional p.e. normativa alemana (Bundesministerium der Justiz und für Verbraucherschutz, 2004). La eficiencia del sistema de biofiltración es comparable con otros estudios donde se han obtenido entre 75 y 97\% de degradación de DQO (Demirel, Yenigun \& Onay, 2005). No obstante, cabe mencionar que procesos físico-químicos carecen de altos rangos de remoción de materia orgánica disuelta en comparación con procesos biológicos (Dawood, Kumar \& Sambi, 2011).

Según las características del agua residual, con un DQO inicial de $10.000 \mathrm{mg}^{-1} \mathrm{~L}^{-1}$ y $\mathrm{DBO}_{5}$ de 4.000 $\mathrm{mg} \cdot \mathrm{L}^{-1}$, la relación $\mathrm{DQO} / \mathrm{DBO}$ fue de 0,4 . Esta relación permitió conocer previamente que los contaminantes del agua residual tenían capacidad de biodegradación. La presencia de compuestos químicos sanitizantes en el agua residual serían los responsables de altos valores de DQO en comparación con la presencia de carbohidratos (lactosa), proteínas y lípidos de la leche. Debido a la inyección de aire al sistema, los cambios de pH en el rango ácido, la degradación de materia orgánica disuelta se logró mediante catabolismo oxidativo a dióxido de carbono. Microorganismos quimioorganoheterótrofos formadores de biopelículas utilizan compuestos orgánicos como fuente de carbono y de energía a través de glicólisis aerobia y ciclo de ácidos tricarboxílicos, con producción de dióxido de carbono. 


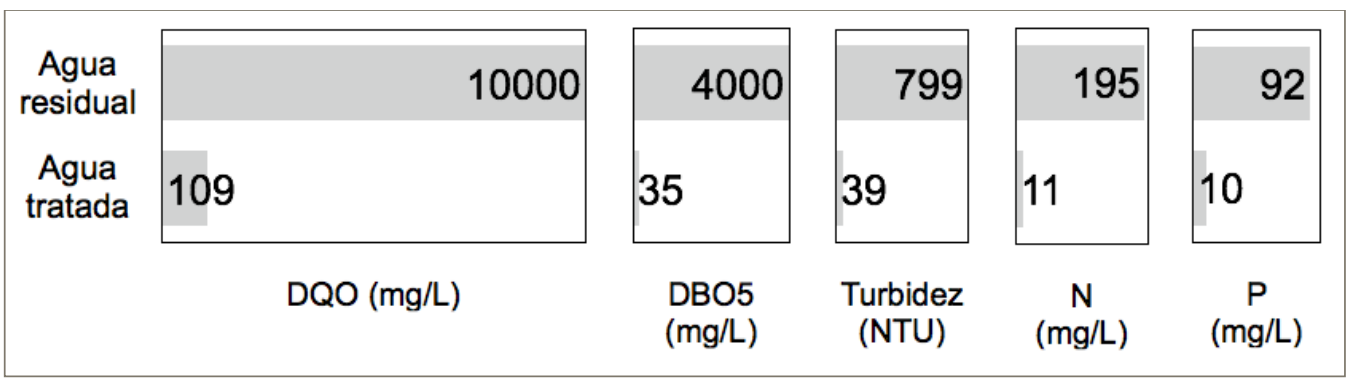

Figura 7. Calidad físico-química y biológica del agua residual y tratada.

\section{Conclusiones y Recomendaciones}

Se realizó la biodegradación de contenido orgánico del agua residual proveniente de una industria láctea generando una remoción considerable de turbidez en función del cambio del pH, temperatura y tiempo de operación. La acidez final del medio indica la presencia de ácidos orgánicos que no fueron completamente degradados en la biofiltración, pero en la sedimentación secundaria, los ácidos orgánicos remanentes tuvieron respuesta positiva frente a la zeolita para una floculación natural con niveles de remoción final de turbidez de hasta 95,2\%. La degradación de la materia orgánica fue aerobia a través de un lecho con biopelículas en tres filtros percoladores conectados en serie. Esta degradación alcanzó porcentajes de 98,9\% en demanda química y $99,1 \%$ en demanda bioquímica de oxígeno. Se utilizaron microorganismos aislados de la misma muestra de agua residual de una planta procesadora de leche ubicada en San Miguel de Nono (Ecuador).

Para futuros estudios, se recomienda un monitoreo continuo del nivel de degradación de ácidos grasos, una caracterización microbiana en biopelículas, así como evaluación de condiciones de sobrevivencia microbiana bajo efectos cambiantes de temperatura y desecación. En conclusión, de acuerdo a los resultados experimentales del presente estudio, para tratamientos aerobios de agua residual de lecheras mediante biofiltros, la presencia de grasas no impide una remediación óptima sino que promueve incrementos de la reducción de turbidez y neutralización del pH. Con el presente trabajo se promueve la implementación de soluciones de bajo coste y accesibilidad tecnológica para las industrias lácteas.

\section{Bibliografía}

Bundesministerium der Justiz und für Verbraucherschutz. (2004). Verordnung über Anforderungen an das Einleiten von Abwasser in Gewässer (Abwasserverordnung - AbwV) Anhang 3 Milchverarbeitung. Retrieved from http://www.gesetze-im-internet.de/abwv/anhang_3.html

Buntner, D., Sánchez, A., \& Garrido, J. M. (2013). Feasibility of combined UASB and MBR system in dairy wastewater treatment at ambient temperatures. Chemical Engineering Journal, 230, 475-481. 
Carvalho, F., Prazeres, A. R., \& Rivas, J. (2013). Cheese whey wastewater: Characterization and treatment. Science of The Total Environment, 445-446(0), 385-396.

Daverey, A., \& Pakshirajan, K. (2015). Treatment of dairy wastewater containing high amount of fats and oils using a yeast-bioreactor system under batch, fed-batch and continuous operation. Desalination and Water Treatment, 1-7.

Dawood, A. T., Kumar, A., \& Sambi, S. S. (2011). Study on anaerobic treatment of synthetic milk wastewater under variable experimental conditions. International Journal of Environmental Science and Development, 2(1), 17-23.

De Mes, T. Z. D., Stams, A. J. M., Reith, J. H., \& Zeeman, G. (2003). Methane production by anaerobic digestion of wastewater and solid wastes. Bio-Methane \& Bio-Hydrogen.

Demirel, B., Yenigun, O., \& Onay, T. T. (2005). Anaerobic treatment of dairy wastewaters: a review. Process Biochemistry, 40(8), 2583-2595.

Food and Agriculture Organization of the United Nations. (2014). Milk production. Dairy production and products. Retrieved from http://www.fao.org/agriculture/dairy-gateway/milk-production

Karadag, D., Koroglu, O. E., Ozkaya, B., Cakmakci, M., Heaven, S., Banks, C., \& Serna-Maza, A. (2015). Anaerobic granular reactors for the treatment of dairy wastewater: A review. International Journal of Dairy Technology, 68(4), 459-470.

Månsson, H. L. (2008). Fatty acids in bovine milk fat. Food \& Nutrition Research, 52(0).

Ministerio de Industrias y Productividad. (2014). La industria lechera busca generar mayor valor agregado para sumarse al Cambio de la Matriz Productiva. Retrieved from http://www.industrias.gob.ec/bp-072-la-industria-lechera-busca-generar-mayor-valoragregado-para-sumarse-al-cambio-de-la-matriz-productiva/

Ministerio del Ambiente. (2008). Texto Unificado de Legislación Secundaria del Ministerio del Ambiente. Libro VI, Anexo 1: Norma de calidad ambiental y de descarga de efluentes: recurso agua. Ministerio del Ambiente, Ecuador.

Naz, I., Saroj, D. P., Mumtaz, S., Ali, N., \& Ahmed, S. (2015). Assessment of biological trickling filter systems with various packing materials for improved wastewater treatment. Environmental Technology, 36(4), 424-434.

Porwal, H. J., Mane, A. V., \& Velhal, S. G. (2015). Biodegradation of dairy effluent by using microbial isolates obtained from activated sludge. Water Resources and Industry, 9, 1-15. 
R Core Team. (2014). R: A language and environment for statistical computing. R Foundation for Statistical Computing, Vienna, Austria. Retrieved from http://www.Rproject.org/

Russell, V. L. (2014). Response-Surface Methods in R, Using rsm. Retrieved from http://www.jstatsoft.org/v32/i07/

Sirianuntapiboon, S., Jeeyachok, N., \& Larplai, R. (2005). Sequencing batch reactor biofilm system for treatment of milk industry wastewater. Journal of Environmental Management, 76(2), 177-183.

Verheijen, L. (1996). Management of Waste from Animal Product Processing. Food and Agriculture Organization. Retrieved from http://www.fao.org/wairdocs/lead/x6114e/x6114e06.htm 